\title{
Fibromyalgia-Associated Hyperalgesia and Psychopathological Alterations are Unrelated to Gut Microbiome Diversity
}

Thomas Weber ( $\nabla$ webertom2002@yahoo.de )

Medical University of Graz

Eva Tatzl

Medical University of Graz

Karl Kashofer

Medical University of Graz

Magdalena Holter

Medical University of Graz

Slave Trajanoski

Medical University of Graz

Andrea Berghold

Medical University of Graz

Akos Heinemann

Medical University of Graz

Peter Holzer

Medical University of Graz

Michael Karl Herbert

Medical University of Graz

\section{Research Article}

Keywords: Fibromyalgia-syndrome (FMS), quantitative sensory testing (QST), FMS patients, QST and psychopathology

Posted Date: April 26th, 2021

DOl: https://doi.org/10.21203/rs.3.rs-434221/v1

License: (c) (1) This work is licensed under a Creative Commons Attribution 4.0 International License.

Read Full License 


\section{Abstract}

Fibromyalgia-syndrome (FMS) is a complex disease characterized by chronic widespread pain and additional symptoms including depression, cognitive dysfunction ("fibro-fog") and maldigestion. We examined whether FMS-related pain parameters assessed by quantitative sensory testing (QST) are related to changes of microbial diversity.

We recruited 25 patients with FMS and 26 age- and sex-matched healthy controls. Medical background, food habits, psychopathology and quality of life were assessed with questionnaires. Stool samples were analyzed by $16 S$ rRNA gene amplification and sequencing. QST was performed according to the protocol of the German Network for Neuropathic Pain.

QST showed that both lemniscal and spinothalamic afferent pathways are altered in FMS patients relative to healthy controls and that peripheral as well as central pain sensitization processes are manifest. Psychometric assessment revealed enhanced scores of depression, anxiety and stress. In contrast, neither the composition nor the alpha- and beta-diversity of the fecal microbiome was changed in FMS patients.

FMS patients segregate from healthy controls in various parameters of QST and psychopathology, but not in terms of composition and diversity of the fecal microbiome. Despite consideration of several confounding factors we conclude that the impact of the gut microbiome on the pathophysiology of FMS is limited.

\section{Introduction}

Patients suffering from chronic pain create a total socioeconomic burden of around 3,000 Euro per year. ${ }^{1}$ Fibromyalgia-syndrome (FMS), a chronic pain disease with a high incidence, affects about $2-4.7 \%$ of the population worldwide. ${ }^{2}$

Patients with FMS suffer from various symptoms: most common is chronic widespread pain in different body areas. Moreover, various kinds of additional symptoms can occur, such as fatigue, cognitive difficulties ("fibro-fog"), sleep disturbances, and depression. A high percentage of patients also has to deal with maldigestion. For example, irritable bowel syndrome is very common in FMS. ${ }^{2-6}$

The pathophysiology of FMS appears very complex and is hardly understood. The International Association for the Study of Pain (IASP) has recently introduced the ICD-11 term of "nociplastic pain" 7 as a new designation to describe pain in the absence of actual or threatened tissue damage. FMS is classified as a disease in which nociplastic pain plays a crucial role in the pathophysiology. 2,8,9

It has been proposed that both genetic and environmental predisposition might play a role, including bacterial infections (eg Borrelia) or a stressful life event (eg loss of partner). ${ }^{10,11}$ It is not clear whether peripheral inflammation (small fiber neuropathy) or central nociplastic changes catalyze chronic 
widespread pain. ${ }^{12-14}$ Probably due to an interplay of peripheral and central mechanisms, neuromorphological changes are brought about that trigger further symptoms. Changes at spinal and supraspinal levels can also lead to reduced endogenous pain inhibition. ${ }^{15}$ Furthermore, significant alterations of the autonomic nervous system occur, which may explain many of the FMS-specific symptoms. ${ }^{8,16}$ For instance, Furlan et al. ${ }^{17}$ showed that patients with FMS present with enhanced cardiovascular sympathetic activity, although there are currently controversies about basal levels of sympathetic activity. ${ }^{18}$ Other studies ${ }^{19}$ showed even lower sympathetic levels in FMS.

Recently, the gut microbiota has been considered to be another factor relevant to the pathophysiology of FMS $^{20,21}$ Minerbi et al. ${ }^{22}$ and Clos-Garcia et al. ${ }^{23}$ were able to show that specific bacteria of the gut microbiome are changed in patients with FMS. The altered bacteria were mostly gram-negative and thus can shed lipopolysaccharides (LPS) that may become systemic through a leaky gut barrier. Via activation of Toll-like receptor-4 (TLR-4) on monocytes and neutrophil granulocytes, LPS can induce systemic inflammation which leads to neuronal alterations that contribute to central sensitization. ${ }^{20}$

In the studies of Minerbi et al. ${ }^{22}$ and Clos-Garcia et al. ${ }^{23} \mathrm{FMS}$ was diagnosed by a thorough clinical assessment of the patients involving a variety of questionnaires. Since self-reported pain profiles do not always match quantitative sensory testing (QST) parameters in FMS patients ${ }^{24-26}$ the first major objective of the current study was to investigate whether FMS-related profiles of QST parameters are associated with alterations of the fecal microbiome. Given that FMS is linked with several psychological disturbances ${ }^{27}$, the second major objective was to examine whether FMS-related changes in psychometric scores (depression, anxiety, stress) are coupled to changes in microbial composition. In this way we set out to obtain more specific information on the relevance of the gut microbiome to the pathophysiology of FMS.

\section{Methods}

This study was a single-center, case-control study, which was performed at the Clinical Division of Special Anesthesiology, Pain Medicine and Intensive Care at the Medical University of Graz, Austria, from December 2018 until December 2019. A permission of the local ethics committee (Medical University of Graz, Austria) was obtained prior to the beginning of the study (registration number: EK $31-012 \mathrm{ex}$ 18/19). This study was performed according to the Declaration of Helsinki in 2013 and the current STROBE guidelines for reporting observational studies. Informed consent was obtained from all study participants.

We recruited 25 patients with FMS and 26 healthy control (HC) subjects (age- and sex-matched). FMS was diagnosed according to the criteria of the American College of Rheumatology published in $2016^{28}$ (criteria catalogue and physical examination) and FMS symptom-severity was quantitated according to the Patient-Health-Questionnaire-15 (PHQ-15). ${ }^{29}$ The inclusion and exclusion procedure is illustrated in Fig. 1. 
We recruited FMS patients via an invitation letter at the pain clinic of the University Hospital of Graz. Ageand sex-matched voluntary employees at the University Hospital of Graz were asked to join the study as healthy controls. After they signed an informed consent, they had to complete one single study visit. After checking the inclusion- and exclusion criteria, all patients underwent a physical neuro-orthopedic examination to exclude reasons for musculoskeletal pain other than FMS. First, we took blood samples for a separate study. Patients and healthy probands then underwent quantitative sensory testing (QST). Last, we gave detailed information about how to complete several questionnaires and to handle the stool samples. Patients and healthy controls were instructed to fill the stool collection tubes (4 stool collection tubes) at home and then send them to the laboratory with a prepared, cooled package. We included an electronic thermometer for tracking a constant temperature (maximum temperature allowed $+15^{\circ} \mathrm{C}$ for 24 hours) at each parcel. All samples were then immediately frozen at $-70^{\circ} \mathrm{C}$. One stool sample was needed for microbiome analysis, all others were kept for a separate study.

\section{Demographic data}

Demographic data and detailed medication anamnesis were obtained via a structured talk and documented on the German Pain Questionnaire. Furthermore, all study participants were asked to answer the validated German version of the Depression-Anxiety-Stress Scale (DASS-G) ${ }^{30}$, the ICD-10 SymptomRating Brief Description (ISR-10) ${ }^{31}$ and the Marburg Questionnaire for Quality of Life (MbFhW). ${ }^{32}$

Patients and $\mathrm{HC}$ subjects also had to complete a questionnaire about their dietary habits. This questionnaire was derived from the Food-Frequency-Questionnaire of the Robert-Koch-Institute and asks about the most important dietary habits (12 items) for a balanced diet. ${ }^{33}$

\section{Microbiome analysis}

Stool samples were collected in Stool Collection Tubes with DNA Stabilizer (Stratec Molecular, Berlin, Germany) and then frozen at $-80^{\circ} \mathrm{C}$. Bacterial DNA was extracted with the Maxwell RSC Blood DNA Kit (Promega, Mannheim, Germany) according to the manufacturer's instructions with slight modifications for stool samples. The stool samples were homogenized with lysis buffer on a MagNA Lyser Instrument using MagNA Lyser Green Beads (Roche Diagnostics GmbH, Mannheim, Germany). The samples were treated then with $2.5 \mathrm{mg} / \mathrm{ml}$ lysozyme (Roth $\mathrm{GmbH}$, Karlsruhe, Germany) for $30 \mathrm{~min}$ at $37^{\circ} \mathrm{C}$ followed by digestion with $1 \mathrm{mg} / \mathrm{ml}$ proteinase $\mathrm{K}$ for $60 \mathrm{~min}$ at $56^{\circ} \mathrm{C}$. The enzyme was inactivated at $95^{\circ} \mathrm{C}$ for $10 \mathrm{~min}$. For the DNA isolation in the Maxwell RSC, $600 \mu$ l of lysate was taken. The concentration of DNA was determined by Picogreen fluorescence. Then, the variable V4 region of the bacterial 16S rRNA gene was amplified using the Mastermix 16s Complete PCR Kit (Molzym, Bremen, Germany) according to the manufacturer's instructions from 20 ng DNA using oligonucleotide primers 16s_515_fwd:

TGCCAGCAGCCGCGGTAA and 16s_806_rev: GGACTACCAGGGTATCTAAT. Afterwards PCR products were subjected to agarose gel electrophoresis and the band of the expected length (350 nt) was excised from the gel and purified using the QiaQick (Qiagen, Hilden, Germany) gel extraction system. The amplicon DNA concentration was measured by Picogreen fluorescence. 
Amplicons from 30 samples were pooled equimolarly and subjected to emulsion PCR in the lon Chef ${ }^{\text {TM }}$ Instrument according to the manufacturer's protocols using the lon 400BP workflow and the lon $530^{\mathrm{Tm}}$ Chip Kit. Sequencing reactions were performed on the lon GeneStudio S5 System running for 1000 flows (all reagents from Thermo Fisher Scientific, MA, USA). The sequence files were analyzed with GALAXY using the QIIME 2019.7 workflow. ${ }^{34-38}$

\section{Quantitative sensory testing (QST)}

QST was carried out according to the protocol of the German Network for the Treatment of Neuropathic Pain (DFNS) ${ }^{39}$ on the non-dominant hand. To measure heat and cold sensory levels and heat and cold pain threshold, we used PATHWAY (Medoc, Ramat Yishai, Israel). Vibration detection threshold was measured with VSA-3000 (Medoc, Ramat Yishai, Israel), pressure detection threshold with Force dial FDK/FDN series (Wagner, Greenwich, CT, USA), mechanical-tactile threshold with von Frey filaments (MARSTOCKnervtest, Marburg, Germany), and dynamic-mechanical sensory threshold with Pinprick (MRC Systems, Heidelberg, Germany). Patients and healthy controls were always tested by the same examiner at the same room temperature, given that the ambient temperature has an influence on pain sensitivity in FMS. $^{40}$

\section{Statistical analysis}

The demographic data were described by mean, standard deviation, median, minimum and maximum as appropriate for continuous variables whereas categorical variables were described by absolute and relative frequencies. The demographic data and nutrition scores of FMS patients and healthy controls were compared by t-test and Fishers exact test.

Statistical analysis was performed with SPSS version $25, \mathrm{IBM} \rightarrow, \mathrm{p}<0.05$ was defined as statistically significant. We used $\mathrm{G}^{*}$ Power $^{41}$ for calculating the number of study participants in FMS and healthy controls. A sample size of 26 in each group will have $80 \%$ power to detect an effect size of 0.8 using a two group t-test with a $5 \%$ two-sided significance level.

For analysis of the human gut microbiome, the Galaxy web platform (http://galaxy.medunigraz.at) ${ }^{37}$ was used. Operational taxonomic units (OTUs) were displayed as OTU tables created with QIIME2 implementation in Galaxy (Version 2019.7) DADA2-based workflow and visualized as principal coordinates analysis (PCOA) plots, and according bar charts were also generated with QIIME2. For the taxonomic classification we used SILVA rRNA database ver 132. Significant differences between FMS and $\mathrm{HC}(\mathrm{p}<0.01)$ were analyzed using the Adonis test, and significant differences in individual bacterial strains were calculated by the Kruskal-Wallis test. Canonical correspondence analysis (CCA) and differential taxa abundance analysis with DeSeq2 were performed in $\mathrm{R} \rightarrow$ according to standard protocols. $^{42}$

Raw data of QST underwent further data preparation. After checking the standard distribution with the Shapiro-Wilk test (values above $p>0.05$ were considered as a normal curve of distribution), we added a 
small constant (+ 0.1; Bartlett-Procedure) according to Rolke et al. ${ }^{39}$ where applicable. Standard logarithmic transformation (In) was done for all values except paradoxical heat sensations, cold and heat pain threshold and vibration detection threshold. Parameters were then compared with ANOVA or t-test. Further data preparation for interpretation of gain or loss of function made it necessary to z-transform all values. For FMS, $z$-scores $\left(z\right.$-score $=\left(x_{\text {single participant }}-\right.$ mean $\left.\left._{\text {norms }}\right) / S_{\text {norms }}\right)$ were calculated and then displayed with Excel 2016, Microsoft $\rightarrow$. Values above 0 indicate a gain of function, values below 0 a loss of function. ${ }^{39}$

\section{Results}

\section{Demographic data and general clinical assessment}

All results regarding demographic data are displayed in Table 1.

There were no significant differences in age, sex and body mass index (BMI) between FMS and HC individuals. However, significant $(p<0.01)$ more patients with FMS were smokers than HC subjects. Thirteen patients presented with mild FMS (<14 points in PHQ-15) while 12 patients were classified as suffering from severe FMS (> 15 points in PHQ-15). Another key point in FMS diagnosis was the assessment of psychopathological profile. All pertinent questionnaires showed a significant difference between the two groups. The absolute scores were clinically relevant and confirmed that the two groups differ with regard to depression, anxiety and stress. Regarding dietary habits, neither the distribution of an omnivore diet, vegetarian diet and vegan diet among FMS patients vs. HC subjects showed significant differences, nor the calculated nutrition score between healthy controls and FMS patients. All study participants completed a questionnaire regarding medication intake within the last 3 months. The results show that the use of various medications was more prevalent in FMS patients than HC subjects. 
Table 1

Demographic data (age, sex, BMl, smoking status) and dietary, medication and psychometric profiles of patients with FMS vs healthy controls.

\begin{tabular}{|c|c|c|c|}
\hline & $\begin{array}{l}\text { FMS } \\
N=25\end{array}$ & $\begin{array}{l}\text { Healthy controls } \\
N=26\end{array}$ & $\mathbf{p}$ \\
\hline \multicolumn{4}{|l|}{ Demographics } \\
\hline Female, n (\%) & $22(88)$ & $21(81)$ & 0.69 \\
\hline Age in years, mean (SD) & $49.8 \pm 8.6$ & $50.0 \pm 8.0$ & 0.91 \\
\hline 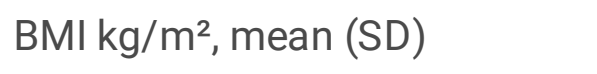 & $25.6 \pm 5.6$ & $23.8 \pm 4.0$ & 0.11 \\
\hline Smoker, n (\%) & $6(24)$ & - & $<0.01$ \\
\hline \multicolumn{4}{|l|}{ Diet } \\
\hline Omnivore, n (\%) & $17(68)$ & $21(84)$ & \\
\hline Vegetarian, n (\%) & $7(28)$ & $5(16)$ & \\
\hline Vegan, n (\%) & $1(4)$ & - & \\
\hline Nutrition Score, mean (SD) & $5.52 \pm 1.39$ & $4.32 \pm 1.49$ & 0.78 \\
\hline \multicolumn{4}{|l|}{ Medications } \\
\hline NSAR, n (\%) & $17(68)$ & $8(31)$ & 0.17 \\
\hline Antidepressant, n (\%) & $9(36)$ & $2(8)$ & $<0.01$ \\
\hline Antihypertensive Drugs, n (\%) & $5(20)$ & $2(8)$ & 0.12 \\
\hline PPI, n (\%) & $6(24)$ & $2(8)$ & 0.01 \\
\hline Antibiotics, n (\%) & $3(12)$ & - & 0.20 \\
\hline THC/CBD, n (\%) & $10(40)$ & - & $<0.01$ \\
\hline \multicolumn{4}{|l|}{ Psychometric questionnaires } \\
\hline Depression, Median (IQR) & $6.90(8.00)$ & $1.25(1.00)$ & $<0.01$ \\
\hline Anxiety, Median (IQR) & $7.39(5.00)$ & $1.25(1.75)$ & $<0.01$ \\
\hline Stress, Median (IQR) & $11.20(8.00)$ & $2.45(3.00)$ & $<0.01$ \\
\hline Symptom Rating, Median (IQR) & $1.06(0.84)$ & $0.30(0.31)$ & $<0.01$ \\
\hline Quality of Life, Median (IQR) & $16.80(13.00)$ & $37.64(6.00)$ & $<0.01$ \\
\hline
\end{tabular}

Diet: Number of patients with FMS vs healthy controls under an omnivore, vegetarian or vegan diet. Adapted nutrition score (by the German Food Frequency Score) in FMS patients vs healthy controls. Medication taken by the study participants on a regular basis for at least 3 months before the study visit. 
Psychometric scores assessed by DASS (Depression-Anxiety-Stress-Score) with subresults of D (Depression), A (Anxiety) and S (Stress), ISR-10 (ICD-10-Symptom-Rating) and MbFhW (Marburg Questionnaire for Quality of Life) in FMS patients vs healthy controls. The values shown are means, with $\mathrm{SD}$ or IQR given in parenthesis. Fishers exact test was used to calculate the $\mathrm{p}$-values shown above.

Abbreviations: BMI - body mass index, NSAID - non-steroidal anti-inflammatory drug, PPI - proton pump inhibitor, THC - tetrahydrocannabinol, CBD - cannabidiol

\section{Microbiome analysis}

Stool samples were sequenced with a total of 3,491,933 reads and an average of $68,838 \pm 73,362$ reads per sample. Detailed analysis showed that the Shannon alpha-diversity, Evenness vector, Faith's phylogenetic diversity and observed OTUs (Table 2) did not differ between the two groups.

Table 2

Alpha-diversity statistics for the microbiome in FMS patients vs. healthy controls.

\begin{tabular}{|llll|}
\hline & FMS & Healthy controls & $\mathbf{p}$ \\
\hline OTUs & $194.85(42.98)$ & $197.99(49.69)$ & 0.81 \\
\hline Faith-PD & $16.07(2.71)$ & $16.13(2.98)$ & 0.95 \\
\hline Evenness vector & $0.73(0.05)$ & $0.73(0.05)$ & 0.96 \\
\hline Shannon & $5.58(0.56)$ & $5.58(0.56)$ & 0.92 \\
\hline
\end{tabular}

Table 2: Alpha-diversity statistics for the microbiome in FMS patients vs. healthy controls. The values shown are means, with SD given in parenthesis. P-values were calculated with Kruskal-Wallis Test.

Abbreviations: OTU -operational taxonomic unit, Faith PD - Faith's phylogenetic diversity.

The distribution of bacterial taxa in FMS patients and healthy controls is displayed in Fig. 2. Although subtle differences in the distribution of bacterial taxa between the two groups were observed, these differences were not statistically significant (Table 3).

Table 3

Statistical analysis of microbial beta-diversity in FMS patients vs healthy controls

\begin{tabular}{|llll|}
\hline & FMS & Healthy Controls & p \\
\hline Bray-Curtis & $0.99 \pm 0.01$ & $0.99 \pm 0.02$ & 0.238 \\
\hline Jaccard distance & $1.0 \pm 0.01$ & $0.99 \pm 0.02$ & 0.357 \\
\hline Unweighted unifracture & $0.15 \pm 0.03$ & $0.15 \pm 0.03$ & 0.259 \\
\hline Weighted unifracture & $0.53 \pm 0.11$ & $0.52 \pm 0.12$ & 0.425 \\
\hline
\end{tabular}


The values shown are means with SD. ANOVA was used to calculate p-values.

Canonical correspondence analysis (CCA) of the bacterial composition in FMS patients and healthy controls was also unable to disclose a significant difference (Fig. 3). Furthermore, differential taxa abundance analysis with DESeq2 likewise failed to reveal clinically relevant significant differences of the bacterial strains (see supplementary data).

\section{Quantitative Sensory Testing (QST)}

QST was able to disclose significant differences in the cold pain threshold $(p<0.05)$, pressure pain threshold $(p<0.001)$, vibration detection threshold $(p<0.05)$, mechanical detection threshold $(p<0.05)$, mechanical pain threshold $(p<0.001)$ and dynamic mechanical allodynia $(p<0.001)$ between FMS patients and $\mathrm{HC}$ subjects (Table 4). To set all variables measured in the two populations in relation, we calculated z-values and display them in Fig. 4.

Table 4

Results of quantitative sensory testing of FMS patients vs healthy controls ( $\mathrm{HC}$, raw data). The values shown are means, with SD given in parenthesis; * indicates a significant p-value, ANOVA was used as a statistical test.

\begin{tabular}{|c|c|c|c|}
\hline QST parameter & FMS & $\mathrm{HC}$ & $\mathrm{p}$ \\
\hline $\mathrm{CDT}{ }^{\circ} \mathrm{C}$ & $30.82(0.77)$ & $30.59(0.76)$ & 0.08 \\
\hline WDT ${ }^{\circ} \mathrm{C}$ & $34.26(1.50)$ & $34.22(1.17)$ & 0.85 \\
\hline $\mathrm{CPT}{ }^{\circ} \mathrm{C}^{\star}$ & $18.15(8.91)$ & 9.09 (6.71) & 0.001 \\
\hline $\mathrm{HPT}{ }^{\circ} \mathrm{C}$ & $41.06(3.46)$ & $42.02(3.15)$ & 0.51 \\
\hline $\mathrm{TSL}{ }^{\circ} \mathrm{C}$ & $33.44(1.41)$ & $33.06(1.21)$ & 0.44 \\
\hline MDT mN & $0.99(0.69)$ & $1.16(0.74)$ & 0.48 \\
\hline MPT mN* & $32.94(36.28)$ & $49.82(51.63)$ & 0.04 \\
\hline MPS PR* & $5.78(8.95)$ & $1.10(2.15)$ & 0.001 \\
\hline DMA PR* & $1.52(1.36)$ & $0.16(1.29)$ & 0.002 \\
\hline PHS n & $0.12(0.44)$ & $0(0)$ & 0.15 \\
\hline WUR PR & 3.45 (3.38) & $2.80(1.17)$ & 0.91 \\
\hline PPT kPa* & $2.08(0.66)$ & $2.91(1.13)$ & 0.003 \\
\hline VDT $\mu m^{*}$ & $0.82(0.74)$ & $0.66(0.47)$ & 0.04 \\
\hline
\end{tabular}

Abbreviations: $\mathrm{CDT}=$ cold detection threshold, $\mathrm{WDT}=$ warm detection threshold, $\mathrm{CPT}=$ cold pain threshold, HPT = heat pain threshold, TSL = temperature sensory limen, MDT = mechanical detection threshold, MPT = mechanical pain threshold, MPS = mechanical pain sensitivity, DMA = dynamic 
mechanical allodynia, $\mathrm{PHS}=$ paradoxical heat sensations, $\mathrm{WUR}=$ wind up ratio, $\mathrm{PPT}=$ pressure pain threshold, VDT = vibration detection threshold

\section{Discussion}

The results of this study show that patients suffering from FMS present with multiple changes in pain modalities as assessed by QST, which go along with enhanced scores of stress, depression and anxiety. These findings attest to complex alterations in pain sensitivity in the periphery and pain processing in the central nervous system, that appear to be interrelated with disturbances of emotion, affect and stress resilience. The present results failed, however, to confirm recent reports that changes in the gut microbiota may contribute to the symptoms of FMS. We were unable to find any significant differences in the composition and diversity of the gut microbiota as analyzed in stool samples of FMS patients vs healthy controls.

The results of QST imply that both peripheral and central sensitization processes contribute to the symptoms of FMS. The lower thresholds (see Table 4) for thermal and mechanical stimuli (CPT, MDT, MPT) together with allodynia (DMA) prove the contribution of unmyelinated $\mathrm{C}$-fibers and $\mathrm{A} \beta$-fibers to pain sensitization. Üceyler et al. ${ }^{13}$ suggested that a small fiber neuropathy might contribute to chronic widespread pain in FMS. We were able to show that sensory conduction by $A \beta$ - fibers in the lemniscal pain pathway (fine touch, vibration, proprioception) is altered in FMS. Especially dynamic mechanical allodynia and vibration detection threshold (DMA, VDT) show a gain of function. As a conclusion, peripheral sensitization seems to make an important contribution to chronic widespread pain, but on the basis of these findings it stays unknown whether additional central sensitization develops as a result of altered peripheral input or vice-versa. ${ }^{43}$

Central pain sensitization is one key domain in FMS. Furthermore, the psychopathological profile of FMS patients may contribute to the symptoms of $\mathrm{FMS}^{27}$. The psychopathological profile may, on the one hand, contribute to enhanced pain sensation while, on the other hand, chronic pain may give rise to anxiety, depression and stress. ${ }^{44}$ Our data clearly show that psychological stress but also the anxiety, depression and symptom severity scores are elevated in patients with FMS vs. HC subjects (DASS-S 11.2 vs. 2.45) and their quality of life is impaired. Stress has been reported to impair central pain control in FMS patients. ${ }^{45}$ In addition, enhanced sympathetic nerve activity has an adverse impact on pain intensity in FMS patients..$^{8,17}$ Our findings are in line with reports that psychopathological alterations of the anxious-depressive-type are highly prevalent in FMS patients and that these psychological disturbances may lower the capacity to cope with the disease. ${ }^{27}$

Given that the gut microbiota has been proposed to impact on pain processing in the brain ${ }^{20,46,47}$ and this has also been reported for FMS, ${ }^{22,23}$ we set out to investigate whether distinct changes disclosed by QST and psychometric evaluation could be associated to distinct changes in gut microbiome composition. However, our analysis of FMS patients vs. healthy controls failed to disclose any significant alterations in 
the profile of the fecal microbiome as assessed by Shannon alpha-diversity and various beta-diversity indices (Bray-Curtis, observed OTUs, evenness vector, Faith's phylogenetic diversity, Jaccard distance, unweighted and weighted unifracture, canonical correspondence analysis, and differential gene expression analysis). We therefore conclude that, in the FMS patients studied here, the sensory und psychometric alterations cannot be attributed to alterations in fecal microbiome composition. This finding is different from those of Minerbi et al. ${ }^{22}$ and Clos-Garcia et al. ${ }^{23}$ who reported FMS-related differences especially in the beta-diversity of the fecal microbiome and identified a number of bacterial taxa that were up- or down-regulated. However, they also noted that the overall population structure and diversity of the microbiome in FMS patients was relatively similar to those in healthy reference subjects.

There are several confounding factors that could explain why our findings differ from those of Minerbi et al. ${ }^{22}$ and Clos-Garcia et al. ${ }^{23}$.

Although we cannot dismiss that analysis of the microbiome at higher resolution could disclose some subtle FMS-related differences, we rather favor other reasons, given that the gut microbiome is under the influence of many internal and external factors. Thus, the psychopathology of the FMS patients studied here could have an impact, given that various neuropsychiatric disorders including depression are associated with a disturbance of the gut microbiome. ${ }^{48,49}$ The DASS-D, DASS-A and DASS-S scores in FMS patients were all significantly higher than those in healthy controls, which is likely to be associated with increased activity in the autonomic nervous system and the hypothalamic-pituitary-adrenal axis. Another issue to be considered is that in our study the patients were allowed to take routine medication up the study visit. It is known that, apart from antibiotics, many drugs including NSAIDs ${ }^{50}$, antidepressant drugs $^{51}$ and proton pump inhibitors ${ }^{52}$ significantly alter the profile of the gastrointestinal microbiome. Furthermore, antidepressant drugs and analgesic medicine are well known to influence sensitive parameter in QST (eg CPT). Dietary habits are among the major determinants of microbial structure and diversity in the gut ${ }^{53}$, but this factor may be of minor relevance to the present study because dietary habits and nutrition scores were similar in FMS patients and healthy controls. Taken these considerations together, it is reasonable to suspect that FMS-related alterations in the fecal microbiome may be obscured by the influence which psychopathology, medication and/or diet have on the microbiome. This contention is in line with the conclusion of Erdrich et al. ${ }^{54}$ that the relationship between the gut microbiome and the pathophysiology of FMS remains a largely underexplored area.

We also need to mention some limitations of the current study. First, the number of study participants (25 FMS vs $26 \mathrm{HC}$ ) was relatively small with a high drop-out rate around $20 \%$. Second, we do not know whether the collection of stool in tubes with preserving fluid impacts on the analysis relatively to other sampling methods. Third, the stool samples of two FMS patients could not be used for microbiome analysis due to the low quality of the PCR results. Fourth, we included 3 patients that recorded regular intake of antibiotics within the last three months. Of course, this might lead to a huge bias in microbiome analysis. All three confirmed that they completed the antibiotic treatment at least 10 days before they 
took the stool samples. Fifth, unattended lifestyle factors such as exercise may have had a relevant impact on gut microbiome.

\section{Declarations}

\section{Data availability statement}

The datasets generated during and/or analysed during the current study are available from the corresponding author on reasonable request.

\section{Acknowledgements}

We thank the City of Graz, the Austrian Society for Anaesthesiology and Spectrum Therapeutics Austria and for financial support. Publication fees were reimbursed from the Doctoral School Sustainable Health Research of the Medical University of Graz.

\section{Author Contributions}

WT substantial contributions to the conception, design of the work, acquisition, analysis and interpretation of data

TE acquisition, analysis and substantively revised manuscript

KK acquisition, analysis

HM acquisition, analysis and substantively revised manuscript

TS acquisition, analysis

BA acquisition, analysis and substantively revised manuscript

HA substantial contributions to the conception, substantively revised manuscript

HP interpretation of data, drafted the work and substantively revised it

HMK substantial contributions to the conception and design of the work

\section{Additional Information}

\section{Competing interests}

The author(s) declare no competing interests

\section{References}


1. Mayer, S. et al. The societal costs of chronic pain and its determinants: The case of Austria. PLoS One. 14, 1-18 (2019).

2. Sarzi-Puttini, P., Giorgi, V., Marotto, D. \& Atzeni, F. Fibromyalgia: an update on clinical characteristics, aetiopathogenesis and treatment. Nat. Rev. Rheumatol. 16, 645-660 (2020).

3. Johnson, C. M. \& Makai, G. E. H. Fibromyalgia and Irritable Bowel Syndrome in Female Pelvic Pain. Semin. Reprod. Med. 36, 136-142 (2018).

4. Lachaine, J. \& Beauchemin, C. L. P. Clinical and economic characteristics of patients with fibromyalgia syndrome. Clin J Pain. 26, 284-290 (2010).

5. Mathieu, N. Comorbidités somatiques dans le Syndrome de l'Intestin Irritable: fibromyalgie, syndrome de fatigue chronique et cystite interstitielle/syndrome de la vessie douloureuse. Gastroenterol. Clin. Biol. 33, S17-S25 (2009).

6. Rossi, A. et al. Fibromyalgia and nutrition: What news? Clin. Exp. Rheumatol. 33, S117-S125 (2015).

7. Kosek, E. et al. Do we need a third mechanistic descriptor for chronic pain states? Pain. 157, 13821386 (2016).

8. Zamunér, A. R. et al. Relationship between sympathetic activity and pain intensity in fibromyalgia. Clin. Exp. Rheumatol. 33, S53-S57 (2015).

9. Üçeyler, N. et al. Etiology and pathophysiology of fibromyalgia syndrome: Updated guidelines 2017, overview of systematic review articles and overview of studies on small fiber neuropathy in FMS subgroups. Schmerz. 31, 239-245 (2017).

10. Cassisi, G., Sarzi-Puttini, P. \& Cazzola, M. Chronic widespread pain and fibromyalgia: Could there be some relationship with infections and vaccinations? Clin. Exp. Rheumatol.29, (2011).

11. Sommer, C. et al. Ätiologie und Pathophysiologie des Fibromyalgiesyndroms. Schmerz. 26, 259-267 (2012).

12. Cagnie, B. et al. Central sensitization in fibromyalgia? A systematic review on structural and functional brain MRI. Semin. Arthritis Rheum. 44, 68-75 (2014).

13. Üçeyler, N. et al. Small fibre pathology in patients with fibromyalgia syndrome. Brain. 136, 18571867 (2013).

14. Caro, X. J. \& Winter, E. F. Evidence of abnormal epidermal nerve fiber density in fibromyalgia: Clinical and immunologic implications. Arthritis Rheumatol. 66, 1945-1954 (2014).

15. Dickenson, A. H., Chapman, V. \& Green, G. M. The pharmacology of excitatory and inhibitory amino acid-mediated events in the transmission and modulation of pain in the spinal cord. Gen. Pharmacol. 28, 633-638 (1997).

16. Martinez-Lavin, M. Autonomic Nervous System Dysfunction May Explain the Multisystem Features of Fibromyalgia. Semin. Arthritis Rheum. 29, 197-199 (2000).

17. Furlan, R. et al. Abnormalities of cardiovascular neural control and reduced orthostatic tolerance in patients with primary fibromyalgia. J. Rheumatol. 32, 1787-1793 (2005). 
18. Okifuji, A. \& Turk, D. C. Stress and psychophysiological dysregulation in patients with fibromyalgia syndrome. Appl. Psychophysiol. Biofeedback. 27, 129-141 (2002).

19. del Reyes, G. A. \& de la Coba, P. Reduced activity, reactivity and functionality of the sympathetic nervous system in fibromyalgia: An electrodermal study. PLoS One. 15, 1-14 (2020).

20. Guo, R., Chen, L. H., Xing, C. \& Liu, T. Pain regulation by gut microbiota: molecular mechanisms and therapeutic potential. Br. J. Anaesth. 123, 637-654 (2019).

21. Osadchiy, V., Martin, C. R. \& Mayer, E. A. The Gut-Brain Axis and the Microbiome: Mechanisms and Clinical Implications. Clin Gastroenterol Hepatol. 17, 322-332 (2019).

22. Minerbi, A. et al. Altered microbiome composition in individuals with fibromyalgia. Pain. 160, 25892602 (2019).

23. Clos-Garcia, M. et al. Gut microbiome and serum metabolome analyses identify molecular biomarkers and altered glutamate metabolism in fibromyalgia. EBioMedicine. 46, 499-511 (2019).

24. Tampin, B., Briffa, N. K. \& Slater, H. Self-reported sensory descriptors are associated with quantitative sensory testing parameters in patients with cervical radiculopathy, but not in patients with fibromyalgia. Eur. J. Pain (United Kingdom). 17, 621-633 (2013).

25. Wodehouse, T. et al. A pilot study investigating whether quantitative sensory testing alters after treatment in patients with fibromyalgia. Br. J. Pain. 12, 250-256 (2018).

26. Pickering, G. et al. Electrochemical Skin Conductance and Quantitative Sensory Testing on Fibromyalgia. Pain Pract. 20, 348-356 (2020).

27. Garcia-Fontanals, A. et al. Vulnerability to Psychopathology and Dimensions of Personality in Patients With Fibromyalgia. Clin J Pain. 33, 991-997 (2017).

28. Wolfe, F. et al. 2016 Revisions to the 2010/2011 fibromyalgia diagnostic criteria. Semin. Arthritis Rheum. 46, 319-329 (2016).

29. Häuser, W., Brähler, E., Wolfe, F. \& Henningsen, P. Patient Health Questionnaire 15 as a generic measure of severity in fibromyalgia syndrome: Surveys with patients of three different settings. $J$. Psychosom. Res. 76, 307-311 (2014).

30. Nilges, P. \& Essau, C. Die Depressions-Angst-Stress-Skalen: Der DASS - ein Screeningverfahren nicht nur für Schmerzpatienten. Obere Extrem. 10, 649-657 (2015).

31. Tritt, K., Von Heymann, F., Zaudig, M., Zacharias, I. \& Söllner, W. Development of the 'ICD-10Symptom-Rating'(ISR) questionnaire. Z Psychosom Med Psychother. 54, 409-418 (2008).

32. Basler, H. D. Marburger Fragebogen zum habituellen Wohlbefinden: Untersuchung an Patienten mit chronischem Schmerz. Schmerz. 13, 385-391 (1999).

33. Haftenberger, M. et al. Relative validation of a food frequency questionnaire for national health and nutrition monitoring. Nutr. J. 9, 1-9 (2010).

34. Schmieder, R. \& Edwards, R. Fast identification and removal of sequence contamination from genomic and metagenomic datasets.PLoS One6, (2011). 
35. Bragg, L., Stone, G., Imelfort, M., Hugenholtz, P. \& Tyson, G. W. Fast, accurate error-correction of amplicon pyrosequences using Acacia. Nat. Methods. 9, 425-426 (2012).

36. Edgar, R. C. Search and clustering orders of magnitude faster than BLAST. Bioinformatics. 26, 24602461 (2010).

37. Afgan, E. et al. The Galaxy platform for accessible, reproducible and collaborative biomedical analyses: 2018 update. Nucleic Acids Res. 46, W537-W544 (2018).

38. McDonald, D. et al. The Biological Observation Matrix (BIOM) format or: how I learned to stop worrying and love the ome-ome. Gigascience. 1, 7 (2012).

39. Rolke, R. et al. Quantitative sensory testing in the German Research Network on Neuropathic Pain (DFNS): Standardized protocol and reference values. Pain. 123, 231-243 (2006).

40. Berwick, R. J., Siew, S., Andersson, D. A. \& Marshall, A. Systematic Review Into the Influence of Temperature on Fibromyalgia Pain: Meteorological Studies and Quantitative Sensory Testing. $J$ Pain. 6, S1526-5900 (2021).

41. Faul, F., Erdfelder, E., Lang, A. G. \& Buchner, A. G*Power 3: A flexible statistical power analysis program for the social, behavioral, and biomedical sciences. Behav. Res. Methods. 39, 175-191 (2007).

42. Varet, H., Brillet-Guéguen, L., Coppée, J. Y., Dillies, M. A. \& SARTools A DESeq2- and edgeR-based R pipeline for comprehensive differential analysis of RNA-Seq data. PLoS One. 11, 1-8 (2016).

43. Bourne, S. \& Machado, A. G. Basic anatomy and physiology of pain pathways. Neurosurg Clin N Am. 25, 629-638 (2014).

44. Duque, L. \& Fricchione, G. Fibromyalgia and its New Lessons for Neuropsychiatry. Med. Sci. Monit. Basic Res. 25, 169-178 (2019).

45. Coppieters, I. et al. Effects of stress and relaxation on central pain modulation in chronic whiplash and fibromyalgia patients compared to healthy controls. Pain Physician. 19, 119-130 (2016).

46. Boer, C. G. et al. Intestinal microbiome composition and its relation to joint pain and inflammation. Nat. Commun. 10, 1-9 (2019).

47. Minerbi, A. \& Fitzcharles, M. A. Gut microbiome: pertinence in fibromyalgia. Clin. Exp. Rheumatol. 38, 99-104 (2020).

48. Kelly, J. R., Keane, V. O., Cryan, J. F. \& Clarke, G. Mood and Microbes: Gut to Brain Communication in Depression. Gastroenterol Clin North Am. 48, 389-405 (2019).

49. Morais, L. H., Schreiber, H. L. \& Mazmanian, S. K. The gut microbiota-brain axis in behaviour and brain disorders. Nat. Rev. Microbiol. https://doi.org/10.1038/s41579-020-00460-0 (2020).

50. Rogers, M. A. M. \& Aronoff, D. M. The influence of non-steroidal anti-inflammatory drugs on the gut microbiome. Clin. Microbiol. Infect. 22, 178.e1-178.e9(2016).

51. Lukić, I. et al. Antidepressants affect gut microbiota and Ruminococcus flavefaciens is able to abolish their effects on depressive-like behavior. Transl. Psychiatry9, (2019).

52. Imhann, F. et al. Proton pump inhibitors affect the gut microbiome. Gut. 65, 740-748 (2016). 
53. Zmora, N., Suez, J. \& Elinav, E. You are what you eat: diet, health and the gut microbiota. Nat. Rev. Gastroenterol. Hepatol. 16, 35-56 (2019).

54. Erdrich, S., Hawrelak, J. A., Myers, S. P. \& Harnett, J. E. Determining the association between fibromyalgia, the gut microbiome and its biomarkers: A systematic review. BMC Musculoskelet. Disord. 21, 1-12 (2020).

\section{Figures}

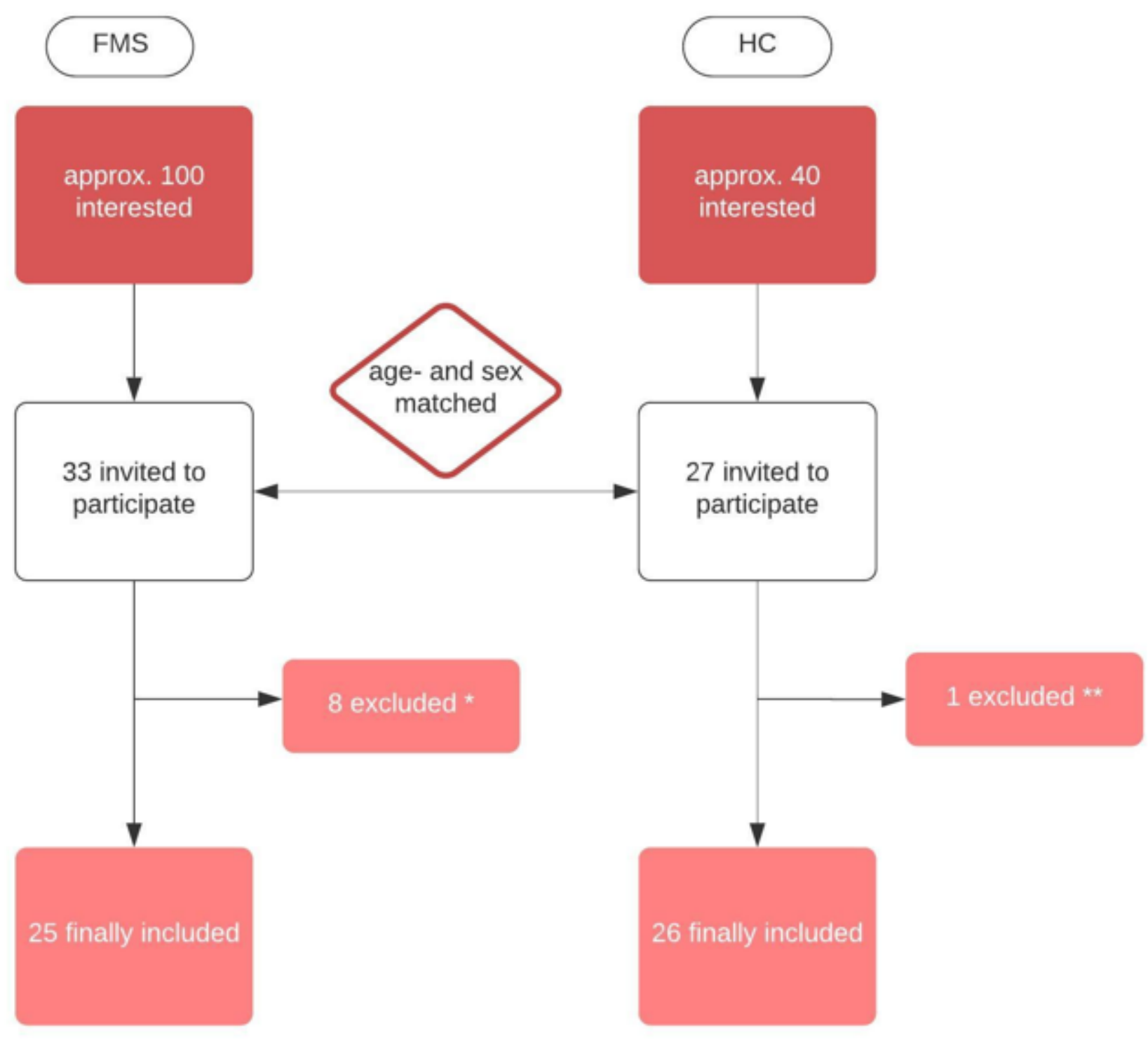

\section{Figure 1}

Flow chart of how many study participants were included/excluded in the study. * 8 were excluded in the FMS-group: 1 Ehlers-Danlos syndrome, 1 unclear autoimmune disease, 1 stroke in anamnesis, 1 acute severe pneumonia, 2 non-compliant, 2 lost contact. $* \star 1$ was excluded in the healthy control (HC) group: autoimmune disease 


\section{Fibromyalgia-Syndrome}

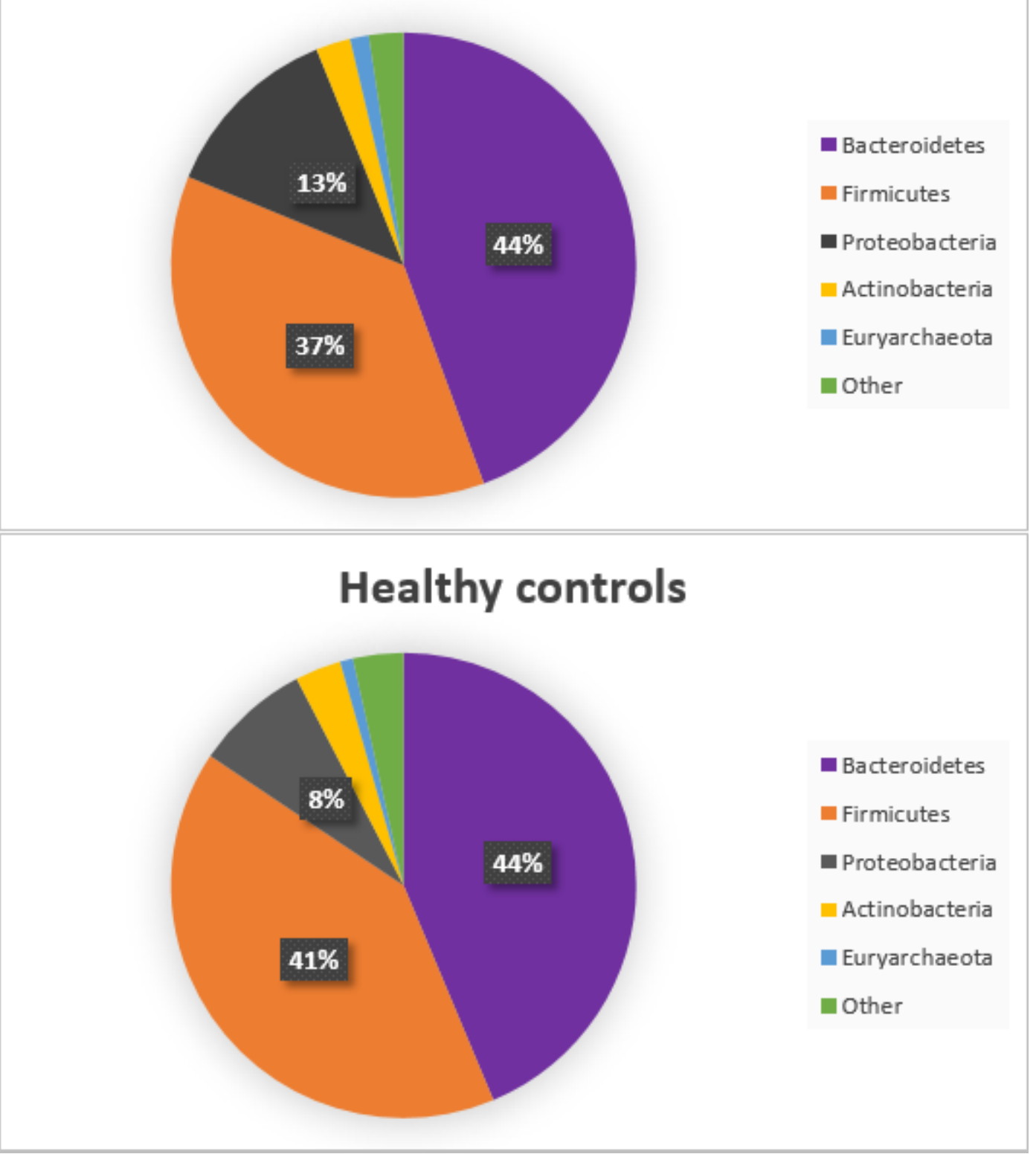

Figure 2

Taxonomic composition of bacterial phyla in FMS patients and healthy controls. In FMS, mostly dominant were Bacteroidetes (44\%), followed by Firmicutes (37\%) and Proteobacteria (13\%). For healthy controls, the predominant phyla were Bacteroidetes (44\%), Firmicutes (41\%) and Proteobacteria (8\%). 
Biplot CCA - scaling 1

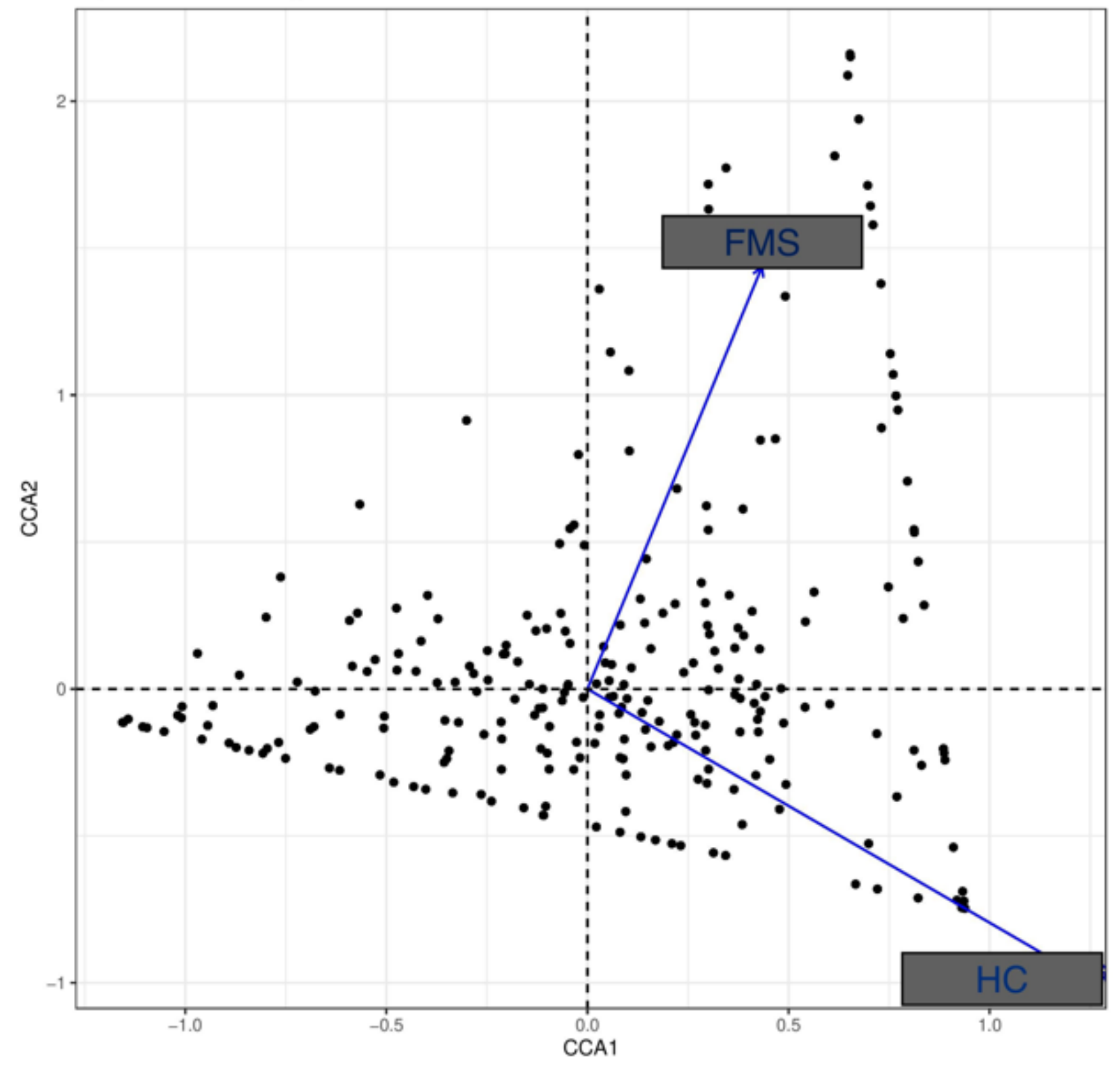

Figure 3

Canonical correspondence analysis of the microbiota in FMS patients and healthy controls $(\mathrm{HC})$, which failed to reveal a distinct "cluster" to each group. 


\section{QST}

\section{5}

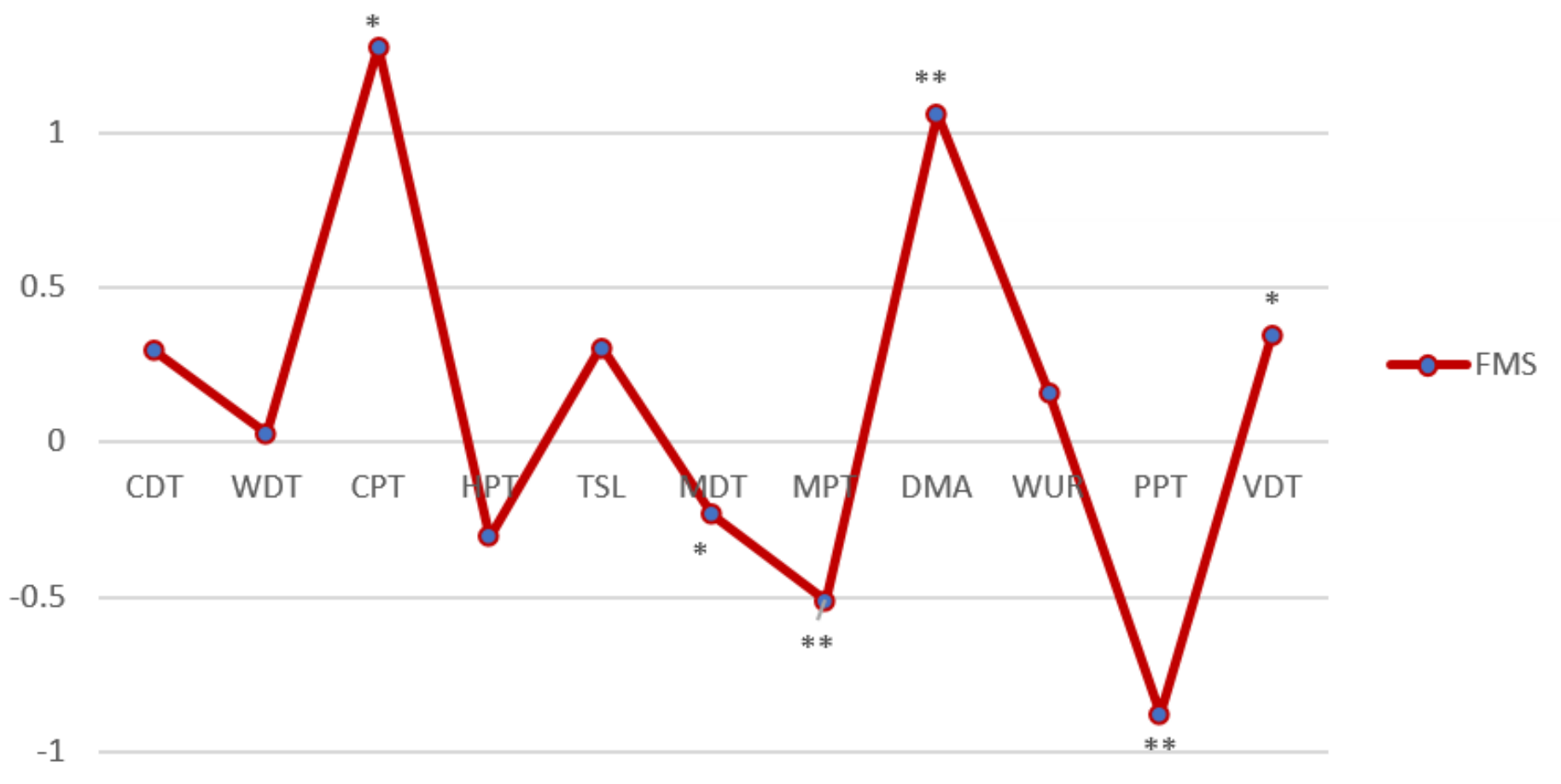

Figure 4

Z-adjusted values of the variables measured in quantitative sensory testing (QST) in FMS patients relative to healthy controls. ${ }^{*} p<0.05,{ }^{* \star} p<0.001$; we used ANOVA for statistical testing. CDT $=$ cold detection threshold, WDT=warm detection threshold, $\mathrm{CPT}=$ cold pain threshold, HPT=heat pain threshold, TSL=temperature sensory limen, MDT=mechanical detection threshold, MPT=mechanical pain threshold, DMA=dynamic mechanical allodynia, WUR=wind up ratio, PPT=pressure pain threshold, VDT=vibration detection threshold

\section{Supplementary Files}

This is a list of supplementary files associated with this preprint. Click to download.

- DESeq2SupplementalMaterialVs2.xlsx

- SupplementalMaterialMethods.pdf 